\title{
Response to comment on: Davidson MB (2007) The dilemma of self-monitoring of blood glucose. Diabetologia 50:497-499
}

\author{
M. B. Davidson
}

Received: 28 March 2007 / Accepted: 2 April 2007 / Published online: 1 May 2007

(C) Springer-Verlag 2007

To the Editor: After several long discussions at meetings concerning self-monitoring of blood glucose (SMBG) in diabetic patients not taking insulin, A. J. Karter and I agree to disagree. I will respond briefly to the points raised in his letter [1].

As stated in my Commentary [2], observational studies can be compromised by self-selection and issues of confounding. Although a few of them have shown a benefit of SMBG in terms of glycaemic control, most have not [3]. Regarding the 'select' randomised clinical trials (RCTs), five of the nine did not find an association, and serious design flaws in the remaining four invalidated (in my view) the conclusion that SMBG per se was responsible for the lowered glycaemia. Although the two meta-analyses found overall changes in $\mathrm{HbA}_{1 \mathrm{c}}$ of $-0.3 \%$ [4] and $-0.4 \%$ [5] in those that performed $\mathrm{SMBG}$, the authors stated that the methodological quality of the studies was limited [4] and that there was either a moderate or high risk of bias in the studies reviewed [5].

The reference [6] citing the importance of behavioural interventions deals mainly with the untranslatability of positive, tightly controlled efficacy trials into messier, realworld effectiveness studies. One problem with this argument is that, to date, the efficacy trials of SMBG in diabetic patients not taking insulin have produced negative results. Based on the overwhelming evidence that lowering glycaemia ameliorates the microvascular complications of diabetes, we first need to demonstrate this outcome before dealing with the valid issues that impede its translation.

\footnotetext{
M. B. Davidson $(\square)$

Clinical Center for Research Excellence,

Charles R. Drew University,

1731 East 120th Street,

Los Angeles, CA 90059, USA

e-mail: mayerdavidson@cdrewu.edu
}

I do agree that SMBG values have no direct effect on glycaemia [1]; appropriate action leading to better control based on these results is necessary. However, as pointed out previously [2], immediate actions in patients not taking insulin are limited and longer term behavioural changes have, for the most part, not materialised. The two references cited to imply that performing SMBG may enhance the ability of patients to make appropriate behavioural decisions $[7,8]$ may not be particularly germane. In one [7], which included patients who did and did not take insulin, changes in $\mathrm{HbA}_{1 \mathrm{c}}$ were $-0.13 \%$ in those performing SMBG after receiving intensive instruction, $-0.04 \%$ in those performing SMBG after receiving standard instruction, and $+0.04 \%$ in those receiving standard care with no new instruction in SMBG at the start of the study. Significantly more patients in the first group than in the other two groups saw improvements in their $\mathrm{HbA}_{1 \mathrm{c}}$ level. However, as Gertrude Stein is alleged to have said, 'A difference to be different must make a difference.' That would certainly not seem to be the case here since this difference would not lead to any change in patient outcomes.

In the second study the intervention patients were taught how to use the internet and uploaded their SMBG values at their convenience while the control patients simply visited the outpatient department every 3 months (as did the intervention group) [8]. Most patients were not taking insulin. Regarding the point raised about intensive nurse case management [1], the 40 intervention patients in this study were serviced by three endocrinologists (a professor and two clinical instructors), a nurse and a dietitian. The two clinical instructors logged on to the system daily and sent appropriate recommendations every 2 weeks (based on the Staged Diabetes Management Protocols, with any changes in medication cleared by the professor). Approximately half of the patients logged on to the system more 
than 3 times per week. The mean $\mathrm{HbA}_{1 \mathrm{c}}$ levels throughout the 30 month study were significantly lower in the intervention group $(6.9 \%)$ than in the control group $(7.5 \%)$. As discussed in their letter, translating this approach into the real world does not seem very practical.

We can only hope that the long-term behavioural changes secondary to SMBG are much better than those achieved in response to self-monitoring of body weight with weighing scales since, to date, there is no evidence of this. Failure of sustained weight loss is rife in our society, despite frequent interactions with scales. In the meantime, we continue to spend over half a billion dollars per year (in the USA) on SMBG in diabetic patients not taking insulin while waiting for the Godot of better control. Rather than shunt aside a proven clinical approach to improving diabetes outcomes [9-11], we should work on finding ways to cost-effectively incorporate nurse management into our present (dysfunctional) system of caring for people with diabetes.

Duality of interest None declared.

\section{References}

1. Kolb H, Martin S, Karter AJ (2007) Comment on: Davidson MB (2007) The dilemma of self-monitoring of blood glucose. Diabetologia 50:497-499, Diabetologia DOI 10.1007/s00125007-0693-1
2. Davidson MB (2007) The dilemma of self-monitoring of blood glucose. Diabetologia 50:497-499

3. Davidson MB (2005) Counterpoint: Self-monitoring of blood glucose in type 2 diabetes not receiving insulin: a waste of money. Diabetes Care 28:1531-1533

4. Welschen LMC, Bloemendal E, Nijpels G et al (2005) Selfmonitoring of blood glucose in patients with type 2 diabetes who are not using insulin: a systematic review. Diabetes Care 28:1510-1517

5. Sarol JN Jr, Nicodemus NA Jr, Tan KM et al (2005) Selfmonitoring of blood glucose as part of a multi-component therapy among non-insulin requiring type 2 diabetes patients: a metaanalysis (1996-2004) Curr Med Res Opin 21:173-184

6. Glasgow RE, Lichtenstein E, Marcus AC (2003) Why don't we see more translation of health promotion research to practice? Rethinking the efficacy-to-effectiveness transition. Am J Public Health 93:1261-1267

7. Moreland EC, Volkening LK, Lawlor MT et al (2006) Use of a blood glucose monitoring manual to enhance monitoring adherence in adults with diabetes: a randomized controlled trial. Arch Intern Med 166:689-695

8. Cho JH, Chang SA, Kwon HS et al (2006) Long term effect of the Internet-based glucose monitoring system on $\mathrm{HbA}_{1 \mathrm{c}}$ reduction and glucose stability: a 30-month follow-up study for diabetes management with a ubiquitous medical care system. Diabetes Care 29:2625-2631

9. Davidson MB, Castellanos M, Duran P et al (2006) Effective diabetes care by a registered nurse following treatment algorithms in a minority population. Am J Manag Care 12:226-232

10. Shojana KG, Ranji SR, McDonald KM et al (2006) Effects of quality improvement strategies for type 2 diabetes on glycemic control: a meta-regression analysis. JAMA 296:427-440

11. Davidson MB, Ansari A (2007) Effect of a nurse-directed diabetes disease management program on emergency room and urgent care visits and hospitalizations in a minority population. Diabetes Care $30: 224-227$ 